\title{
Archipiélagos de la novela inglesa: los islotes de Eliza Haywood y Daniel Defoe en el surgimiento de un género moderno
}

\section{Archipelagos of the English Novel: Bridging the Islands of Haywood and Defoe}

\author{
Gabriela VILLANUEVA-NORIEGA \\ Facultad de Filosofía y Letras, \\ Universidad Nacional Autónoma de México \\ México
}

\begin{abstract}
Resumen:
El artículo hace una introducción comparativa a la obra del escritor inglés Daniel Defoe y de la novelista Eliza Haywood, de la misma nacionalidad, a la vez que examina el papel que ambos autores desempeñaron en el desarrollo de la novela inglesa a lo largo del siglo XVIII y las tradiciones que se desprenden de ésta. Para establecer el punto de contacto entre estas dos formas de narrar, el artículo se centra en la relación entre cuerpo, control y escritura y las formas disímiles en que ambos autores representan la experiencia del cuerpo a través de la narrativa.
\end{abstract}

Palabras clave: novela inglesa, literatura del siglo XVIII, crítica literaria, narrativas, realismo, corporalidad, géneros literarios, novela rosa, novela de aventuras, escritura y género

\begin{abstract}
:
This paper introduces the works of Daniel Defoe and Eliza Haywood in a comparative manner while it examines each author's role in the development of the English Novel during the eighteenth century and its legacy. To establish a point of contact between these two very different narrative styles, the paper focuses on the connection between body, writing, and control, and the dissimilar ways in which both authors convey bodily experience by means of narrative.
\end{abstract}

Keywords: English novel, eighteenth-century literature, literary criticism, written narratives, Realism, body studies, literary genres, romance, gendered writing 
El interés de Virginia Woolf en la novela del siglo XVIII, patente en sus ensayos con motivo del bicentenario de la publicación de Robinson Crusoe, así como en sus lecturas de Laurence Sterne y sus referencias, escuetas y un tanto desencantadas, sobre Eliza Haywood, parecen relacionarse estrechamente con el programa de renovación de la novela que los escritores y las escritoras habían emprendido al despuntar el siglo Xx. En su texto sobre la novela de Defoe, Woolf hace un examen sobre las formas en las que quienes escriben producen mundos acorde a las maneras cambiantes en que éstos se perciben. Así, explica que:

\begin{abstract}
It would seem to be true that people who live cheek by jowl and breathe the same air vary enormously in their sense of proportion; to one the human being is vast, the tree minute; to the other, trees are huge and human beings insignificant little objects in the background. So, in spite of the text-books, writers may live at the same time and see nothing the same size. (Woolf, 2015)
\end{abstract}

Mediante estas palabras incluidas en su breve ensayo sobre Robinson Crusoe, Woolf da cuenta de la manera en que las novelistas más hábiles nos fuerzan a treparnos momentáneamente sobre sus hombros y nos obligan a mirar el mundo a través de sus ojos hasta que nos orillan a entender la manera en la que jerarquizan los objetos del mundo (Woolf, 2015).

Las formas en las que Eliza Haywood y Daniel Defoe dan orden a sus universos ficticios son a menudo tan disímiles que tratar de esbozar el mapa de un archipiélago que dé sentido al quehacer literario de ambos parece una tarea ardua y, sin embargo, necesaria para revalorar el surgimiento de lo que hoy denominamos la novela moderna en la Inglaterra del siglo XVIII. El interés en celebrar el tricentenario de la publicación de dos obras tan distintas del siglo XVIII en el coloquio "De náufragos emprendedores y pasiones desbordadas: 300 años de Robinson Crusoe (1719), de Daniel Defoe, y Love in Excess (1719), de Eliza Haywood", a principios de 2019, fue justamente poner de relieve el legado de Haywood frente al del afamado Defoe en el muy debatido y cuestionado "surgimiento de la novela" del siglo XVIII, en la medida en que la escritora representa una tradición fundamental, aunque históricamente negada, en la construcción del artefacto llamado novela: a saber, la de la novela sentimental.

Eliza Haywood puede ser considerada como una de las principales artífices del lenguaje en el que se cifraron las gramáticas sentimentales del cuerpo para la imaginación de las lectoras del siglo XVIII en Inglaterra. Esta gramática delineada por Haywood sería una pieza fundamental en el desarrollo de la materia ficticia de este periodo y en la conformación de una cadena mucho más larga de novela popular que sobrevive hasta nuestros días en diferentes formatos: la novela erótica, la novela rosa y en las distintas formas comerciales que ambas asumen al paso del tiempo. El nada ingenuo interés por parte de la novelista inglesa en entender los efectos que tenía el amor sobre los cuerpos de los hombres y las mujeres de su 
época permitió que la autora revelara al mundo las terribles consecuencias que podrían derivarse, en particular para el género femenino, cuando una sucumbía demasiado pronto a sus pasiones corporales. Al mismo tiempo, sus esfuerzos por entender el proceso mediante el cual nuestra voluntad cede y trae consigo su propia perdición la llevó a trazar el abecedario del cuerpo sensual y jadeante y sobrescribirlo en el lenguaje de la novela.

En su dedicatoria para Lasselia de 1725, Haywood explicaba que sus intenciones al escribir esta pequeña novela habían sido las siguientes: "only to remind the unthinking Part of the Worlds, how dangerous it is to give way to Passion" (Haywood, 1725: s. p.). Haywood se excusaba por haber atizado con demasiado ímpetu el fuego de las pasiones en algunas de las páginas de su novela a partir de un fin didáctico:

My Design in writing this little Novel [...] will, I hope, excuse the too great Warmth, which may perhaps, appear in some particular Pages; for without the Expression being invigorated in some measure proportionate to the Subject, "twould be impossible for a Reader to be sensible how far it touches him, or how probable it is that he is falling into those Inadvertencies which the Examples I relate wou'd caution him to avoid. (Haywood, 1725: s. p.)

Claro que al igual que con la apología que hacía Defoe del propósito didáctico de su novela Moll Flanders (1722) cuando explicaba que los crímenes de Moll se exponían con el afán de disuadir a los lectores de caer en las garras de una vida de vicio y perdición, es muy probable que Haywood estuviera al tanto de que buena parte del éxito comercial de sus libros se debía al interés por parte del público lector en este tipo de escenas. Sin embargo, también es relevante notar que Haywood tomó el interés, ciertamente redituable, de sus lectores y lectoras en las escenas de pasión para desmantelar con precisión de relojera la irracionalidad del deseo y la fuerza que ejerce sobre nosotros para así comprender plenamente la manera en que los suspiros, los gemidos y los pechos agitados son sordinas efectivas que acallan los murmullos de la razón.

En la exitosa novela de 1719 de Haywood, Love in Excess, una de las primeras escenas de seducción no consumada entre el conde D'Elmont y la protagonista, Amena, se presenta con un énfasis particular en los efectos que tiene el deseo sobre el cuerpo:

'twas now this inconsiderate Lady found herself in the greatest strait she had ever yet been in; all Nature seem'd to favour his Design, the pleasentness of the Place, the silence of the Night, the sweetness of the Air, perfum'd with a thousand various Odors wafted by gentle Breezes from adjacent Gardens compleated the most delightful Scene that ever was, to offer up a Sacrifice of Love; not a breath but she flew wing'd with desire, and sent soft thrilling wishes to the Soul; Cynthia herself, cold as she is reported, assisted in the inspiration and sometimes show with all her brightness, as it 
were to feast their ravish'd Eyes with gazing on each other's Beauty; then veil'd her Beams in Clouds, to give the Lover boldness, and hide the Virgins blushes. (Haywood, 1722: 28)

La sensualidad palpable en el ambiente presenta una escena que conspira con crueldad en contra de la joven Amena y prepara el escenario para este supersticioso - es decir, irracional - sacrificio que es el de renunciar a nuestra identidad ante el altar del amor. La presencia de la luna como elemento que permite revelar la belleza al tiempo que oculta el pudor muestra con intensidad el deseo femenino encarnado en la figura de la joven protagonista. En este ánimo, Haywood continúa su descripción y explica cómo el ambiente cálido había llevado a Amena a vestirse con gran ligereza esa noche y los efectos que esta circunstancia tienen sobre la seducción:

she had only a thin silk Night Gown on, which flying open as he caught her in his Arms, he found her panting Heart beat measures of consent, her heaving Breast swell to be press'd by his, and every Pulse confess a wish to yield; her Spirits all dissolv'd sunk in a Lethargy of Love, her snowy Arms unknowing grasp'd his Neck, her Lips met his half way, and trembled at the touch; in fine, there was but a moment betwixt her and Ruine; when the tread of some body coming hastily down the walk, oblig'd the half bless'd Pair to put a stop to farther Endearments. (Haywood, 1722: 29)

La manera en que Haywood dosifica los efectos de la seducción juega un papel importante en el arco narrativo de la novela y la manera en la que éste se tensa y se distiende. Claro que esta técnica era bien conocida por otros escritores de la época, como por ejemplo por Samuel Richardson en su famosísima Pamela (1740), quien es casi seguro que estuviera al tanto de las estrategias narrativas de su predecesora. Pero hay una diferencia marcada en la construcción de las protagonistas femeninas en ambos autores en la medida en que Richardson, como muchos otros de sus contemporáneos, harán un objeto de deseo de sus personajes; una presa que sucumbe ante los embates de sus depredadores; en tanto que Haywood construye un sujeto deseante que, en contraste con los personajes de Richardson, cae presa, sobre todo, de su propia corporalidad y no de la corporalidad de un agente externo. Claro que Haywood también sitúa socialmente las políticas del género y sus estándares cambiantes para el sujeto femenino al tiempo en que habla con tono moralizante sobre la virtud y la restricción; sin embargo sus descripciones minuciosas en torno a la experiencia de la sexualidad en el cuerpo femenino constituyen un hecho literario marcadamente distinto del de otros escritores contemporáneos a ella.

La atención que pone Haywood en construir una cartografía de los efectos del amor como experiencia física en sus novelas y textos la llevó a explorar el tema desde ángulos variables como el deseo, la venganza, los celos, la infatuación. En su texto ensayístico Reflections on the Various Effects of Love (1726) Haywood ofrece una suerte de catálogo de los distintos disfraces que usa Cupido en sus 
campañas de conquista. Así, por ejemplo, distingue la experiencia familiar del amor, cuando éste ya se conoce, del deseo apenas descubierto, y explica cómo "new Desires play around the Heart and thrill the swelling Veins, and fill deluded Fancy with a thousand gay Ideas of future Hapiness" (19).

El enfoque de Haywood en los efectos imaginativos que tiene el nuevo amor sobre la mente da cuenta de una observación minuciosa de su entorno social en el que probablemente haya sido capaz de percibir este fenómeno en ocasiones distintas. De esta misma manera, Haywood cuenta anécdotas reveladoras sobre la forma en que las jóvenes inglesas experimentaban los embates de la seducción y el rechazo social que podían experimentar quienes sucumbían a ella. Por ejemplo, en el caso de la relación entre Sophiana y Aranthus, que figura como parte de sus Reflections..., Haywood construye una narración un tanto experimental en la que combina la observación ensayística de precisión cuasi científica, con la tradición epistolar para explicar los efectos sicológicos del amor en una sociedad que ejerce una presión a veces insostenible sobre el género femenino. Así va mostrando cómo el amor muchas veces se inscribe sobre los cuerpos femeninos en forma de ropajes desgarrados, pechos desnudos y jadeantes, ojos enrojecidos por el llanto y la asfixia que provoca el dolor.

Asimismo, Haywood también muestra la forma en que el rechazo podía conducir al deseo de destrucción del cuerpo del otro a través de prácticas imaginativas relacionadas con la superstición. Y así describe las formas en las que la impotencia encontraba una válvula de escape en el sometimiento del cuerpo imaginado del amante (representado por una efigie) a quien se podía torcer, doblar, manipular "into a thousand shapes, dislocating in Imagination every Limb [...] sticking it over from head to toe with pins; stabbing it in the eyes with a penknife and gashing it in those parts which in a living body are most tender" (18). Lejos de mostrar la experiencia del amor como un acto pasivo por parte de los sujetos femeninos, Haywood mostraba las múltiples maneras en las que el amor como experiencia vital y social configuraba de manera ineludible y muchas veces violenta, las identidades femeninas de su época.

La destreza de Haywood para representar los efectos físicos del amor sobre el cuerpo de las mujeres, que dicho sea de paso, constituían en buena medida su base lectora, le ganó el título de "the Great Arbitress of Passion" y llevó a un autor a exclamar: "No more of Phoebus, rising vainly boast / Ye tawny sons of a luxuriant Coast! / While our blest Isle is with such Rays replete / Britain shall glow with more than Eastern Heat!" (Luhning, 2009: 101). Esta representación de Haywood en términos que la transforman en entidad exótica es notable en la medida en la que delata la impresión de que la escritura de esta autora corre por un cauce distinto al que convencionalmente se le atribuía a la isla. Lejos de ser una isla sombría y fría, Inglaterra adquiere visos extravagantes y sensuales mediante la presencia de Haywood. La alusión me parece relevante en la medida en que la tradición representada por Haywood y muchas otras escritoras contemporáneas 
de su época, dentro de las que podemos mencionar a Aphra Behn, Penelope Aubin, Delarivière Manley, entre una larga lista (Simpson, 2017), pone en crisis la construcción del estereotipo flemático inglés y apunta a la presencia de otras narrativas que subyacen a la forma de escribir y la materia sobre la que se construye la novela de estas latitudes.

Es decir que si Daniel Defoe y su Robinson Crusoe representan el prototipo del "self-made English man", Eliza Haywood y muchas otras de sus contemporáneas representarían el lado B de esta tradición. Un lado B que corre de manera paralela y que muchas veces será denostado como literatura efímera y de rápido consumo pero que, desde cierto punto de vista atraviesa de manera tangencial la historia de la construcción de la novela para llegar a piezas tan populares como Fifty Shades of Grey (2011) pero también a novelas como Lady Chatterley's Lover (1928) de D. H. Lawrence o Histoire d'O (1954) de Dominique Aury. Ya son más de treinta años desde la publicación del libro de Jane Spencer The Rise of the Woman Novelist (1986) que fue un parteaguas para el estudio de la novela inglesa en la medida en que puso de relieve esta otra tradición como contrapeso a las formas tradicionales en que este tema se había estudiado, particularmente a partir del canónico libro de Ian Watt, The Rise of the Novel (1957). Pese a esto, cabe señalar que dentro del mundo de habla hispana todavía queda mucho trabajo por hacer en la medida en que muy pocas de estas obras han sido traducidas, hecho que genera una visión parcial y sesgada de la forma en la que surgió la novela dentro de la vertiente anglosajona.

El estudio de estas escritoras no sólo ayuda a poner en tela de juicio el relato convencional heredado de la crítica literaria, sino que también permite aproximarse desde otro ángulo a piezas tan fuertemente establecidas y canónicas como $R o$ binson Crusoe. La particular forma en que Defoe construye una narrativa en la que los objetos cotidianos ocupan un espacio de tanta más relevancia que los cuerpos es uno de los temas que recorren y obsesionan a Virginia Woolf en su estudio de la novela de Defoe. También es una de las observaciones sobre las que J. M. Coetzee elabora en su reescritura de la novela de Defoe, Foe, que coincidentemente fue publicada en el mismo año que el libro de Spencer (1986). En la novela de Coetzee se lidia con la forma en que la ficción construye la verosimilitud. Así, la protagonista Susan Barton explica a Crusoe que

The truth that makes your story yours alone, that sets you apart from the old mariner by the fireside spinning yarns of sea-monsters and mermaids, resides in a thousand touches which today may seem of no importance, such as: When you made your needle, (the needle you store in your belt) by what means did you pierce the eye? When you sewed your hat, what did you use for thread? Touches like these will one day persuade your countrymen that it is all true, every word, there was indeed once an island in the middle of the ocean where the wind blew and the gulls cried from from the cliffs and a man named Cruso paced about in his apeskin clothes, scanning the horizon for a sail. (Coetzee, 1986: 18) 
El comentario oblicuo que hace Coetzee a la escritura de Defoe pone de relieve lo que Virginia Woolf está señalando en su propio texto de 1919: la forma en que la tradición de la novela que se desprende de Defoe pone énfasis en la construcción de un mundo hecho de objetos en el que los cuerpos y sus sensaciones ocupan un lugar mucho menos importante. Desde esta perspectiva, los islotes narrativos que generan Haywood y Defoe no podrían ser más diferentes a pesar de haber estado escribiendo en los mismos años. Como explica Woolf, "writers may live at the same time and see nothing the same" (2015). La vida de los sentidos y del cuerpo ocupan un lugar central en Haywood en tanto que en Defoe, o al menos de manera muy palpable en Robinson Crusoe, el cuerpo parece una presencia incómoda que a menudo se soslaya o se empuja francamente hacia el exterior del universo narrado. En esta medida, poner a Defoe en diálogo con las novelas de Haywood permite advertir fenómenos particulares relacionados con el cuerpo y su materialidad que de otra manera podrían pasar inadvertidos.

La primera descripción panorámica que Robinson hace como narrador de su isla, establece lacónicamente sus primeras observaciones:

\begin{abstract}
I found $[\ldots]$ that the Island I was in was barren [...] un-inhabited except by wild Beasts, of whom, however, I saw none. Yet I saw Abundance of Fowls but knew not their Kinds [...] I shot a great Bird which I saw sitting upon a Tree on the Side of a great Wood, I believe it was the first Gun that had been fir'd there since the Creation of the World. I had no sooner fired, than from all Parts of the Wood there arose an innumerable Number of Fowls, of many Sorts, making a confused Screaming and crying, and every one according to his usual Note, but not one of them of any Kind that I knew. (Defoe, 2007: 46)
\end{abstract}

Al llegar a la isla, las descripciones del narrador se centran en lo que Crusoe ve (o lo que no ve, porque no lo reconoce): una isla estéril, desierta, aves que vuelan pero que no sirven de alimento. Justo en el momento en que las aves cantan en una forma en la que, tal vez, habrían podido revelar algo a un alma más poética que la del protagonista, Crusoe corta la observación para concluir únicamente que no reconocía a ninguna de las aves a su alrededor y que, en cualquier caso, no servían de nada.

Varias lectoras, entre las que se incluye Virginia Woolf, han notado esta ausencia de sensualidad en la isla de Crusoe. Como afirma Carol Houlihan Flynn (1990), es posible que en su autosuficiencia Crusoe llegue a extrañar la tinta y la cerveza pero nunca extraña de manera explícita la compañía femenina (64). Para Woolf (2015), en la isla de Crusoe "There are no sunsets and no sunrises; there is no solitude and no soul. There is, on the contrary, staring us full in the face nothing but a large earthenware pot" (s. p.). Y éste es uno de los grandes logros de Defoe; esta conexión entre la escritura, el orden y el control: una vez que se asienta en la isla, Crusoe va dando orden a su mundo a través de la palabra escrita. La isla en sí 
misma parece un espacio vacío, una tierra baldía que Crusoe poco a poco va parcelando y cercando para tenerla bajo control.

Claro que ésta es una de las características más exploradas de la novela: esta relación entre escritura, orden y colonialismo que hace que Robinson figure de manera tan prominente como prototipo del colonialismo inglés. Sin embargo, me interesa ofrecer una lectura a contrapelo de esta idea que se deriva de las corrientes soterradas que traen los elementos que se resisten a ser narrados y ordenados para adquirir un orden estable y tranquilizador. Corrientes relacionadas con las emociones, el cuerpo y el horror que se dejan entrever sutilmente, casi provocadoramente, para después mantenerlas a raya.

Leer las novelas de Haywood a un lado de las de Defoe permiten sintonizar nuestros oídos para advertir los trazos sutiles de ese cuerpo apasionado y fuera de control que Defoe (o tal vez sea Crusoe) constantemente deja entrar a la narración para después expulsarlo tajantemente. Antes de comenzar a escribir su diario, Crusoe dedica un enigmático pasaje a las cosas que podría haber escrito en él si tan sólo hubiese encontrado pluma y papel en sus primeros días en la isla; días de lo que él denomina "Discomposure of mind" en los que el cuerpo, y no el control de la escritura, prevalecen sobre la existencia del personaje. De manera deliberada, Crusoe incluye una entrada apócrifa y burlona en su diario para representar sus primeros días en la isla:

"Sept. the 30th. After I got to Shore and had escaped drowning, instead of being thankful to God for my Deliverance, having first vomited with the great Quantity of salt Water which was gotten into my Stomach, and recovering myself a little, I ran about the Shore, wringing my hands, and beating my Head and Face, exclaiming at my Misery, and crying out, 'I was undone, undone, till, tired and faint, I was forced to lye down on the Ground to repose, but durst not sleep for fear of being devour'd [...]. (Defoe, 2007: 60)

Las acciones descritas por Robinson cifran el lenguaje del cuerpo en un estado descontrolado: el vómito, el movimiento de las manos, la autoconmiseración en las palabras que salen de la boca. Más adelante en este mismo pasaje, Robinson habla de la forma en que se quedaba viendo el horizonte durante horas, imaginando que veía las velas de un barco acercarse, para después descubrir que había sido engañado por sus sentidos y echarse a llorar como un niño. Sin embargo, lo que me parece francamente notable de este pasaje es que se trata de un narración apócrifa en la medida en la que es tan sólo una narración supuesta que realmente no tiene ningún lugar en el recuento "oficial" de sus días en la isla. Si hubiese tenido materiales para escribir, habría escrito algo así, pero finalmente en su diario queda fijada la misma fecha en la que se inscribe un relato radicalmente distinto: 
September 30, 1659. I poor miserable Robinson Crusoe, being shipwreck'd, during a dreadful storm, in the offing, came on shore on this dismal unfortunate island, which I call'd The Island of Despair, all the rest of the Ship's Company being drown'd, and myself almost dead. (Defoe, 2007: 60-61)

La ambivalencia del individuo se desprende de estos dos pasajes paralelos que muestran, por un lado, las acciones descontroladas del personaje y, por otro, las acciones ordenadas del mismo sujeto a través de su pluma: la identidad se vuelve resultado del ordenamiento de la escritura disciplinada. Al igual que en este episodio en el que cuerpo desenfrenado de Robinson se deja ver un momento y se oculta, pareciera que, en la novela, el cuerpo y sus pasiones acechan los bordes de la narrativa, desde donde asoman la cabeza y luego se sumergen.

En este mismo tenor, se podría decir que ese momento de extrañeza que surge del pasaje en el que Robinson escucha una voz que llama y dice "Poor Robinson Crusoe, Where are you? Where have you been? How come you are here?"(Defoe, 2007: 121) y que resulta no ser más que la voz de su perico, Poll, llamando desde lejos; resulta tanto más extraño cuando ponemos en consideración la circunstancia que, a decir de Robinson, hizo que el ave se aprendiera esas frases. Pese a que Crusoe explica su gusto por enseñar a Poll esas palabras, no deja de sorprendernos este momento de desajuste de la imagen de nuestro personaje racional y autosuficiente, que por un momento se sustituye por la de un Robinson desequilibrado y maniático, que se repite a sí mismo hasta el borde de la locura las frases que después repite su perico: "Poor Robinson Crusoe, Where are you? Where have you been? How come you are here?" (121). Todo el asunto se reviste de poca importancia y humor y rápidamente Crusoe continúa su relato dando orden al mundo que lo rodea mediante la tinta de su pluma. Cada día tiene su lugar en el diario, cada objeto va tomando su sitio. La elaboración de velas, vasijas y canastos se narra con un detalle en ocasiones enervante y así las mesas, los botes de tinta, las provisiones y la pólvora se vuelven esos objetos gigantescos que ocupan la mayor parte del espacio en la novela.

Frente a estos objetos, el cuerpo queda disminuido y minimizado por mero contraste. Defoe dedica páginas y páginas de la novela a describir la producción de los objetos más triviales, al menos en apariencia, y sin embargo reduce el relato de la enfermedad que casi lo lleva a la muerte a un par de líneas en las que Crusoe resume días y semanas que transcurren entre temblores corporales y dolores a los que no da gran importancia más allá de los sueños y revelaciones que se derivaron de estos. Al final puede parecer significativo que al calendario de Crusoe, en el cual centra buena parte de su atención, le haga falta un día una vez que abandona su isla.

En el registro escrito y racional de sus días aislado (que dicho sea de paso, es de precisión inverosímil como tantas otras cosas en la isla) Crusoe deja fuera un día completo. $\mathrm{Al}$ tratar de racionalizar qué pudo haber pasado, el personaje con- 
cluye que debió de haber sido uno que perdió durante la enfermedad que casi lo lleva a la muerte. Se trata de un día en el que Robinson no es nada más que un cuerpo al borde de la muerte, un cuerpo adolorido, que gruñe, gime y duerme pero que finalmente queda significativamente excluido de la narración.

En una línea temporal paralela a la que recorre el relato racional de Robinson Crusoe, el cuerpo del personaje flota en el tiempo, junto con todos los demás cuerpos que flotan en la nada que rodea el espacio ordenado de la novela. Esos cuerpos de los otros tripulantes de su embarcación que quedan reducidos a "three of their Hats, one Cap, and two Shoes that were not Fellows" (Defoe, 2007: 41) o junto a la segunda tripulación que se ahoga frente a su isla de la que sólo encuentra el cuerpo de un niño que queda reducido en su descripción a "a seamen's waistcoast, a pair of open-kneed linen drawers, and a blue linen shirt...two pieces of eight and a tobacco pipe" (Defoe, 2007: 159). Parecería ser que la materia corporal que no puede ordenarse ni controlar por medio de la narración tiene que mantenerse a la deriva en los bordes del islote narrativo que es la novela de Defoe.

Aún queda mucho por decir sobre el tratamiento del cuerpo en la novela de Defoe frente a las maneras en las que su contemporánea, Eliza Haywood, lidió con el mismo tema. También se podría agregar a esta lectura las implicaciones que se derivan de las dimensiones que ambos autores otorgan a la corporalidad con respecto a los lugares de enunciación desde los cuales escriben. La representación de vida interior del cuerpo, en particular del cuerpo femenino, llevada a cabo por Haywood sitúa la relevancia de la escritura como una forma de comprensión de las pasiones que habitan a las mujeres de su época y, en esa medida, pone el peso de las pasiones en conflicto con las expectativas de control que se ciernen sobre ellas. De una manera complementaria, la importancia que Defoe da a los objetos del mundo también dice mucho sobre la construcción de un discurso masculinizante de dominación sobre la naturaleza representado de manera marcada por la relación que Crusoe establece con la isla, pero también, de manera más velada, sobre las formas de control escritural que el personaje/narrador ejerce sobre los cuerpos en la novela, incluido el suyo. Estos temas podrán desarrollarse en otra ocasión mientras dejo la imagen de estos dos islotes literarios conviviendo lado a lado sin ninguna otra conexión más que la que se puede advertir cuando se aguza el oído a las corrientes casi imperceptibles que tocan las orillas de ambas costas.

\section{Bibliografía}

Defoe, Daniel. (2007). Robinson Crusoe. Oxford: Oxford World Classics. FLYNN, Carol Houlihan. (1990). The Body in Swift and Defoe (Cambridge Studies in Eighteenth-Century English Literature and Thought). Cambridge: Cambridge University Press. http://doi.org/10.1017/CBO9780511553462 
HaYwood, Eliza. (1722). Love in Excess: Or the Fatal Enquiry, A Novel. In Three Parts. Londres.

HAYwood, Eliza. (1723). Lasselia or the Self-abandoned. Londres.

HaYwood, Eliza. (1726). Reflections on the Various Effects of Love, According to the Contrary Dispositions of the Person on Whom it Operates. Londres.

Luhning, H. R. (2009). "A Crafted Debut: Haywood's Love in Excess and the Literary Marketplace". Lumen, 28, 97-110. https://doi.org/10.7202/1012040ar SimPSON, Kim. (2017). "Naming, Shaming, Reclaiming: The "Incomparable" Eliza Haywood". Persuations Online, 38(1).

Woolf, Virginia. (2015). The Common Reader. Second Series [Libro electrónico]. Adelaide: The University of Adelaide. 